\title{
Silicon quantum computers take shape in Australia
}

\section{Blueprints emerge from centre tasked with creating a practical quantum device.}

\section{BY ELIZABETH GIBNEY}

0 ilicon is at the heart of the multibilliondollar computing industry. Now, efforts to harness the element to build a quantum processor are taking off, thanks to elegant designs from an Australian collaboration.

In July, the Centre for Quantum Computation and Communication Technology, which is based at the University of New South Wales (UNSW) in Sydney, will receive the first instalment of a Aus $\$ 46$-million (US\$33-million) investment. The money comes from government and industry sources whose goal is to create a practical quantum computer.

At an innovation forum in London on 6 May, hosted by Nature and start-up accelerator Entrepreneur First, two physicists from a group at the UNSW pitched a plan to reach that goal. Their audience was a panel of entrepreneurs and scientists, who critiqued ideas for commercializing a range of quantum technologies, including sensors, computer security and a quantum internet as well as quantum computers.

So far, the UNSW team has demonstrated a system with quantum bits, or qubits, only in a single atom. Useful computations will require linking qubits in multiple atoms. But the team's silicon qubits hold their quantum state nearly a million times longer than do systems made from superconducting circuits, a leading alternative, UNSW physicist Guilherme Tosi told participants at the event. This helps the silicon qubits to perform operations with one-sixth of the errors of superconducting circuits.

If the team can pull off this low error rate in a larger system, it would be "quite amazing", said Hartmut Neven, director of engineering at Google and a member of the panel. But he cautioned that in terms of performance, the system is far behind others. The team is aiming

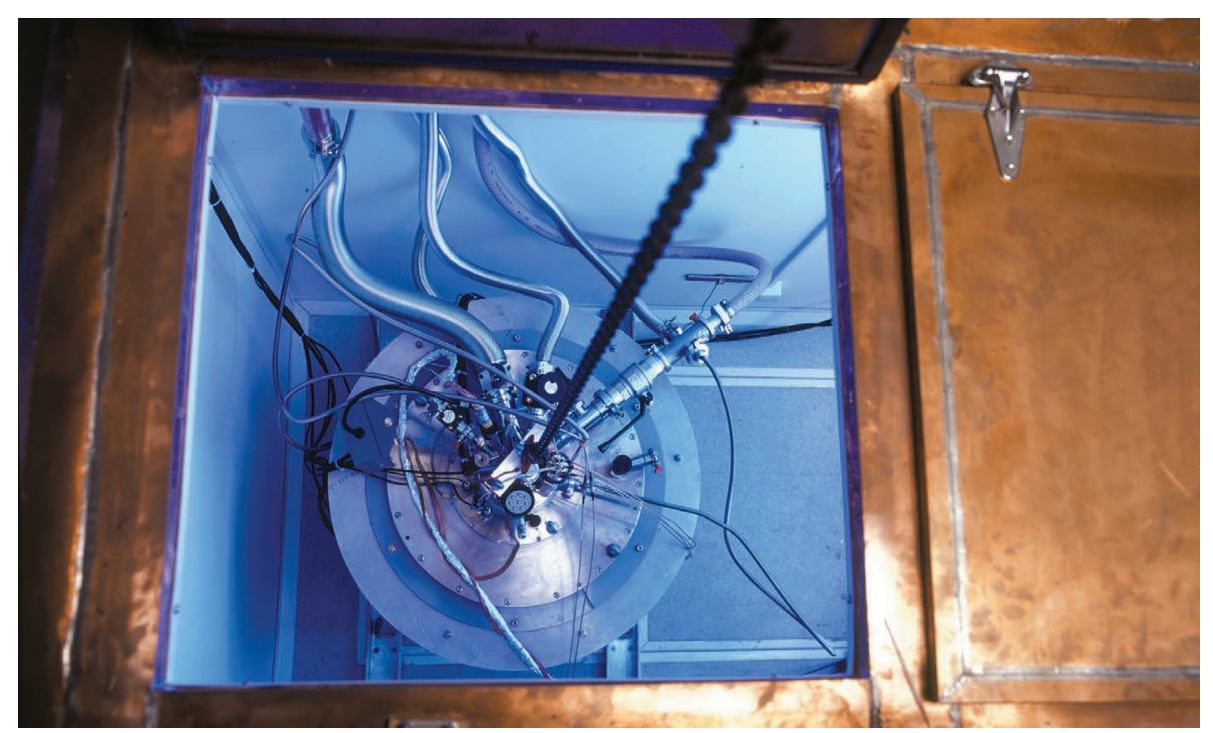

The refrigerator at the University of New South Wales that is used to cool silicon quantum chips.

for ten qubits in five years, but both Google and IBM are already approaching this with superconducting systems. And in five years, Google plans to have ramped up to hundreds of qubits.

A second group from the UNSW has a less robust silicon design that has already demonstrated calculations that link up two qubits, a building block that paves the way for creating more-complex devices.

In a regular computer, each bit can be 'on' or 'off'. In a quantum computer, the qubits can be both on and off at once, which allows them to perform many computations in parallel. This should allow quantum computers to zip through calculations that would take a normal computer longer than the age of the Universe, although the best devices are still much too small to do so.

Silicon is an attractive base for a universal quantum computer - one that can carry out any quantum algorithm - because it is potentially compatible with the microelectronics of existing computers. But silicon systems are still years behind their rivals. One issue has been how to keep delicate quantum states alive for long enough to perform operations.

The scheme showcased at the innovation forum by Tosi and fellow physicist Vivien Schmitt - who are both part of Andrea Morello's lab at the UNSW - addresses this challenge. The qubits are the spins of the electrons and nuclei in phosphorus atoms embedded in a silicon lattice, and are controlled using a special system of electric fields. Because the spins respond only to very specific, tuneable frequencies, they are robust to electrical noise. That allows the qubits to keep their quantum states for one minute and to operate perfectly

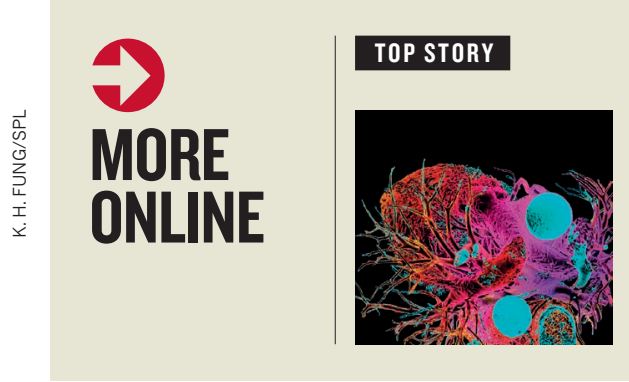

Protective gene offers hope for next blockbuster heart drug go.nature.com/ vclfnl

\section{MORE NEWS}

- Why women earn less: Just two factors explain post-PhD pay gap go.nature.com/jmflm7

- Winners and losers emerge in UK funding shake-up go.nature.com/nta09q - Have physicists found a new force of nature? go.nature.com/69bqyn

\section{NATURE PODCAST}

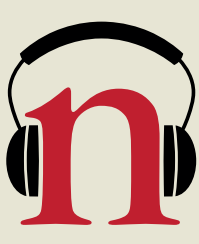

How trees help clouds form; a Neanderthal building project; and comparing meerkats nature. com/nature/podcast 
99.9\% of the time, said Tosi.

Moreover, the electrically controlled qubits can communicate with each other at larger distances than can the qubits in other silicon designs. That bodes well for scaling up because the qubits can be far enough apart to allow room for control and read-out instruments to be placed between them. The atoms also do not need to be placed precisely, so they would fit with existing microprocessor-fabrication techniques, added Tosi.

Although Morello's team has demonstrated the high precision only in a single atom, the researchers have started to experiment with a two-atom system, and expect this level of performance to scale up. "From there, there are no basic first-principle barriers," said Tosi.

He has patented a design for a larger-scale computer and says that it should be possible to manufacture chips that use their system in plants similar to those already used to make present-day microprocessors.

\section{SPIN CITY}

Another silicon project from UNSW, led by physicist Andrew Dzurak, uses as its qubits the spins of electrons in a set-up that is based on modified electrical transistors. Although the qubits are less robust than those in the Morello design, Dzurak's team demonstrated two-qubit calculations last October.

At the London event, Tosi and Schmitt were grilled on the Morello team's plan. "It's a beautiful scheme," said John Morton, an electrical engineer at University College London. But he cautioned that aspects of the business model that the duo presented as part of the event seemed reliant on superconducting qubits not getting any better. "That's a risky thing to do," Morton said. Neven agreed, pointing out that silicon qubits will need to demonstrate advantages in other areas if they are to get a foothold in the future quantum-computer market.

Like superconducting qubits, silicon qubits must be kept at a fraction of a degree above absolute zero. Morton said that the Morello design's big advantage is that the qubits are atomic scale. In principle, that would allow many more to be placed on each chip than is possible with superconducting qubits, which are around 100 micrometres across. To seize this opportunity, the design needs to shed the bulky microwave devices that it proposes as its means to operate between distant qubits, said Neven.

The successes of the Australian quantum centre - which is led by UNSW physicist Michelle Simmons - are partly down to its wider expertise in nanoscale silicon fabrication, says Peter Knight, a physicist at Imperial College London and a panel member at the event. When it comes to fabrication, he says, the centre is way ahead of others working in silicon.

CANCER SCREENING

\section{Myriad Genetics caught in data fight}

\section{Complaint to US government alleges that company violated people's right to access health information.}

\section{BY ERIKA CHECK HAYDEN}

$\checkmark$ enetic-testing firm Myriad Genetics is facing a legal challenge from people who say the company refused to give them access to their own genomic data, in violation of a US government rule on medical records.

Although Myriad has now agreed to release the data to those individuals, the patients are pressing ahead with their complaint to the US government. The skirmish is the latest in a long-running war between Myriad and datasharing advocates, and it could ultimately force the company to provide genetic information that patients could then share with scientists.

The patients, who are represented by the American Civil Liberties Union (ACLU), filed the complaint on 19 May with the US government alleging that Myriad, of Salt Lake City, Utah, had declined to release complete results of tests for the genes BRCA1 and BRCA2. Some variants of these genes are linked to higher risk of cancer; for others, the link to disease is unclear or the variants are considered to be harmless.

Myriad refused to report 'benign' $B R C A$ variants back to patients when they requested this information in February. The ACLU says that the company's denial violates a rule released by the US government in January that gives patients the right to obtain their full lab test results under the Health Insurance Portability and Accountability Act.

\section{TOTAL ACCESS}

Breast-cancer survivor AnneMarie Ciccarella, one of the people who filed the complaint, said that she wants access to her complete data so that she can share it with scientists who are trying to understand the genetic contributions to cancer. "I want to see that the research community has access to every bit of data that has been generated from my body," she said.

On 18 May, after the ACLU announced a press conference to discuss the complaint, Myriad released the data that Ciccarella and her three co-complainants had requested. "We believe the complaint lacks merit and should not be accepted," the company said in a 19 May statement.

Ciccarella and the others who brought the complaint are pressing ahead with their case, in part to set the precedent that companies must legally provide the full results of genetic tests - not release it on a voluntary basis.

Observers say that makes sense, especially given Myriad's history. The company had previously tried to block rivals from providing $B R C A$ tests, asserting that it held patents that gave it the exclusive right to perform such diagnostics.

That changed in June 2013, when the US Supreme Court invalidated Myriad's patents after the ACLU mounted a legal challenge. Myriad has not shared its large database on thousands of $B R C A$ variants, despite requests from researchers studying the genetics of breast cancer. But it may now be forced to provide individual results on

"I want to see
that the research
community
has access to
every bit of data
that has been
generated from
my body."
a patient-by-patient basis if the government decides to accept the latest complaint.

"If I were the plaintiffs, I'd want to make sure the government said that Myriad had to do what it did," says

lawyer and bioethicist Hank Greely at Stanford University in California. "If you're a consumer advocate in the health-care space, Myriad may not be a company you trust."

Heidi Rehm, a geneticist at the non-profit company Partners Healthcare Personalized Medicine in Cambridge, Massachusetts, drafted a statement of support for the complaint against Myriad. She says that as researchers learn more about genetic risks of cancer, they're finding that variants once considered benign might actually contribute to cancer risk.

Rehm and other researchers are pushing for companies and individuals to share their genetic test data with open databases such as ClinVar, and she says that the push for data sharing is gathering increasing momentum. US insurance company Aetna, for instance, has said that it will favour testing companies that deposit data in ClinVar. And the US Food and Drug Administration is considering whether to give companies incentives to deposit their tests results in the database. 